\title{
Transvaginal ultrasound-guided biopsy of adenomyosis
}

\author{
Simone Ferrero ${ }^{1,2}$, Carolina Scala ${ }^{3}$, Valerio Gaetano Vellone ${ }^{4}$, Ennio Biscaldi ${ }^{5}$, Fabio Barra ${ }^{1,2}$ \\ ${ }^{1}$ Academic Unit of Obstetrics and Gynecology, IRCCS Ospedale Policlinico San Martino, Genoa, Italy; ${ }^{2}$ Department of Neurosciences, \\ Rehabilitation, Ophthalmology, Genetics, Maternal and Child Health (DiNOGMI), University of Genoa, Genoa, Italy; ${ }^{3}$ Unit of Obstetrics and \\ Gynecology, Gaslini Institute, Genova, Italy; ${ }^{4}$ Department of Surgical and Diagnostic Sciences, IRCCS Ospedale Policlinico San Martino, Genoa, \\ Italy; ${ }^{5}$ Department of Radiology, Galliera Hospital, Genova, Italy \\ Correspondence to: Simone Ferrero, MD, PhD. Academic Unit of Obstetrics and Gynecology, IRCCS Ospedale Policlinico San Martino, Largo R. \\ Benzi 10, 16132 Genoa, Italy. Email: simone.ferrero@unige.it. \\ Provenance: This is an invited article commissioned by the Section Editor Dr. Hengwei Liu, MD, PhD (Department of Obstetrics and Gynecology, \\ Union Hospital, Tongji Medical College, Huazhong University of Science and Technology, Wuhan, China). \\ Comment on: Tellum T, Qvigstad E, Skovholt EK, et al. In Vivo Adenomyosis Tissue Sampling Using a Transvaginal Ultrasound-guided Core Biopsy \\ Technique for Research Purposes: Safety, Feasibility, and Effectiveness. J Minim Invasive Gynecol 2019;26:1357-62.
}

Submitted Aug 19, 2019. Accepted for publication Sep 18, 2019.

doi: $10.21037 / \mathrm{atm} .2019 .09 .94$

View this article at: http://dx.doi.org/10.21037/atm.2019.09.94

Adenomyosis is a gynecologic disorder characterized by the presence of ectopic endometrial tissue within the uterine myometrium. It can involve most of the myometrium (diffuse adenomyosis), it may be present only in a circumscribed part of the myometrium (focal adenomyosis) or it may appear as a large cyst (adenomyotic cyst). The common sign of adenomyosis is uterine enlargement. Adenomyosis is asymptomatic in approximately one third of patients (1); alternatively, it may cause a wide range of aspecific symptoms including dysmenorrhea, deep dyspareunia and heavy menstrual bleeding $(1,2)$. Adenomyosis may coexist with other gynecological conditions (such as uterine myomas and endometriosis) (3) making its diagnosis even more challenging. In the last 10 years, great attention has been given to the reproductive impact of adenomyosis. It has been demonstrated that women with adenomyosis have increased risk of developing late pregnancy complications caused by placental insufficiency such as small for gestational age newborns, premature rupture of membrane and preeclampsia $(4,5)$.

The definitive diagnosis of adenomyosis is based on the histological examination of the uterus (Figure 1) or of the adenomyotic nodules excised at surgery. There is some debate on the exact histological criteria for the diagnosis, particularly in relation to minimum depth of myometrial infiltration and number of foci required to make the diagnosis. Some pathologists suggest that invasion more than one third of the myometrium is necessary for diagnosing this benign disease; other pathologists propose that a myometrial infiltration greater than $4 \mathrm{~mm}$ is the necessary criteria. Obviously, hysterectomy cannot be performed in women desiring to preserve fertility and surgical excision of focal adenomyosis even when feasible may be technically challenging $(6,7)$. The prevalence of adenomyosis is unknown, because not all patients undergo surgery and until recently there has been a wide variation in the criteria used to perform the ultrasonographic diagnosis of adenomyosis.

Transabdominal ultrasonography has little value in the diagnosis of adenomyosis because it does not allow to discriminate this condition from other myometrial pathologies (such as uterine myomas). The non-invasive diagnosis of adenomyosis can be attempted by different exams (Table 1), among which transvaginal ultrasonography (TVS) and by magnetic resonance imaging (MRI). TVS has several advantages over MRI; in fact, it is performed by the gynecologists and, thus, it is easily accessible, it is well tolerated by the women and it is inexpensive. At TVS, the uterus is often enlarged and this is not caused by the presence of myomas. The traditional ultrasonographic characteristics of adenomyosis are intramyometrial cysts or hyperechoic islands (or both), subendometrial linear striations, ill-defined or irregular junctional zone, asymmetrical thickening of the anterior or posterior myometrial wall, heterogeneous poorly circumscribed areas within the myometrium, anechoic myometrial blood- 


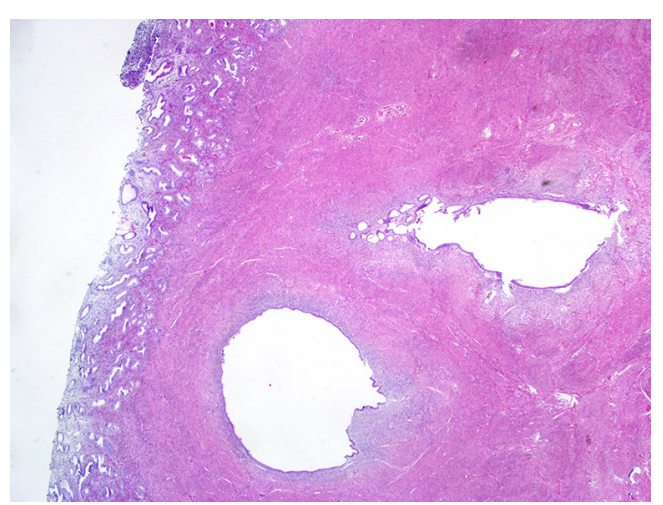

Figure 1 Hysterectomy specimen, hematoxylin and eosin staining, magnification: 20x. Two cystic spaces are lined by endometrial epithelium and stroma deeply embedded in the myometrial wall and overlined by secretive endometrial mucosa.

filled lacunae, fan-shaped shadowing of the myometrium, irregular or interrupted junctional zone (JZ). There is no agreement on which ultrasonographic feature has the highest diagnostic performance. Quite recently, the Morphological Uterus Sonographic Assessment (MUSA) consensus statement standardized the description of the ultrasound findings of adenomyosis (8). Several studies showed that MRI is accurate in the diagnosis of adenomyosis. On T2-weight images the junctional zone myometrium can be clearly differentiated from the endometrium and the outer myometrium; the irregular thickening of this zone is the hallmark of adenomyosis. A systematic review and metanalysis compared the diagnostic performance of TVS and MRI in the diagnosis of adenomyosis (9). Both techniques had high diagnostic performance. TVS had sensitivity of $72 \%$, specificity of $81 \%$, positive likelihood ratio of 3.7 and negative likelihood ratio of 0.3 . MRI had sensitivity of $77 \%$, specificity of $89 \%$, positive likelihood ratio of 6.5 and negative likelihood ratio of 0.2 . Another systematic review and metanalysis including 14 studies (1,895 patients) confirmed that TVS is moderately accurate in diagnosing adenomyosis (10). The pooled sensitivity was $83 \%$, the specificity $85 \%$, the positive likelihood ratio 4.7 and the negative likelihood ratio 0.26 . A recent systematic review and meta-analysis, pooling results from eight studies, showed that two-dimensional TVS has a sensitivity and specificity of $84 \%$ and $64 \%$; for three-dimensional TVS, the sensitivity and specificity are $90 \%$ and $56 \%$, respectively (11).

Asymptomatic adenomyosis does not require treatment.
In symptomatic patients that have completed their reproductive plans, hysterectomy is a potential therapeutic option. Medical therapy should be offered to symptomatic patients who either refuse or wish to postpone surgery. No drug is currently labelled for the treatment of symptoms caused by adenomyosis (12) which is usually managed using the drugs commonly used to treat endometriosis (13-15). The levonorgestrel-releasing intrauterine device is effective in improving heavy uterine bleeding and pain in women with adenomyosis $(16,17)$. Progestins (such as dienogest, norethindrone acetate) and oral contraceptive may improve pain and control heavy menstrual bleeding $(18,19)$. Gonadotropin-releasing hormone analogues can be used in patients not responding to other therapies. Selective progesterone receptor modulators and aromatase inhibitors may be considered for the treatment of adenomyosis in the setting of scientific research. The correct diagnosis of adenomyosis is required for providing the patients adequate counselling and appropriate treatment. For example, it has been shown that the presence of adenomyosis may cause the persistence of pain symptom after surgical excision of deep endometriosis (20).

In the past several authors used various types of biopsies to diagnose adenomyosis. Goswami et al. performed hysteroscopic endomyometrial biopsy to diagnose adenomyosis in 53 patients suffering menorrhagia and in controls who underwent hysterectomy for other indications (such as uterine prolapse) (21). The incidence of adenomyosis by endomyometrial biopsy was $60 \%$ in patients suffering menorrhagia and $33 \%$ in controls. Furthermore, the mean depth of myometrial invasion was $4.1 \mathrm{~mm}$ in patients suffering menorrhagia and $2.0 \mathrm{~mm}$ in the controls.

A prospective non-randomized study assessed the usefulness of laparoscopy-guided myometrial biopsy in the diagnosis of diffuse adenomyosis (22). One hundred patients with suspicion of adenomyosis were included in the study. Ten myometrial biopsy per patient were obtained under laparoscopic guidance using a 14-gauze Tru-cut needle inserted through the abdominal wall. The sensitivity of laparoscopic-guided myometrial biopsies in diagnosing adenomyosis was $98 \%$ (90/92 patients), the specificity $100 \%$, the positive predictive value $100 \%$ and the negative predictive value $80 \%$. The two false negative cases had adenomyosis limited to the inner third of the myometrium.

A retrospective study investigated the role of abdominal ultrasound-guided transvaginal myometrial core needle biopsy (CNB) in the diagnosis of adenomyosis (23). 
Table 1 Main diagnostic methods for the diagnosis of adenomyosis

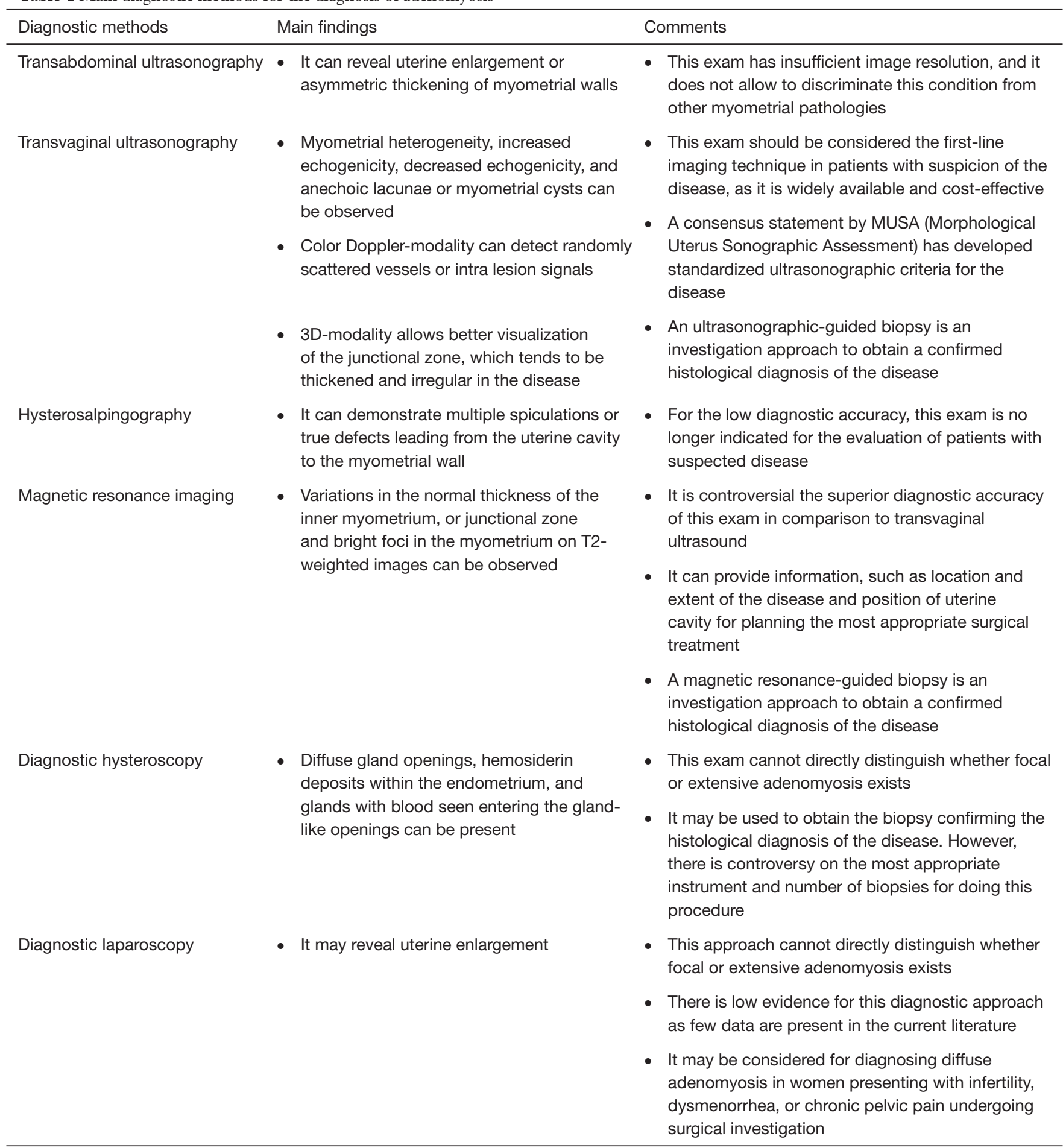


Overall, 1,032 premenopausal women were included in the study; 2,596 CNB were obtained; histology demonstrated adenomyosis in 2,167 . The concordance rate between the CNB and the ultrasonographic diagnosis was $92 \%$. Therefore, the authors concluded that these biopsies can be used for a definitive diagnosis of adenomyosis in patient with clinical or ultrasonographic suspicion who do not undergo hysterectomy.

In a recent prospective cohort study, Tellum et al. (24) investigated whether it is possible to safely obtain adenomyosis tissue in vivo with the aim to use this tissue for molecular studies. The biopsied were performed with the women under general anesthesia before performing hysterectomy. The biopsies were performed transvaginally under ultrasound guidance using a reusable needle guide. The needles had diameter of 14-20 gauge and length of $25-30 \mathrm{~cm}$. In each procedure, the size and length of the needle were chosen depending on the size and characteristics of the uterus. Four biopsies were obtained from each patient. Out of 81 women included in the study, the biopsy was unsuccessful in two patients. In one patient there was a technical problem. In the other patient the biopsy was not performed because of the presence of extensive pelvic adhesions and the risk of bowel perforation. The investigator judged the biopsy easy in $84 \%$ of the cases and difficult in the remaining $15 \%$ of the cases. At laparoscopy, it was observed that the biopsy caused no visible damage in $25 \%$ of the cases, a puncturing of the serosa in $70 \%$, and a minor ongoing bleeding from the small subperitoneal vessels in 5\% (all of which stopped spontaneously during the subsequent surgery). In this series, the amount of bleeding caused by the biopsy was very low (median $2 \mathrm{~mL}$; range, 0-200 mL). Six patients had a blood loss ranging between 50 and $100 \mathrm{~mL} ; 3$ women had blood loss ranging between 150 and $200 \mathrm{~mL}$; all these patients had highly vascularized myometrium at ultrasonography. A small subperitoneal hematoma in the vesicouterine fold was sometimes observed (particularly in women with anteverted uterus). The needle diameter did not influence the amount of bleeding. The $18 \mathrm{G} 25 \mathrm{~cm}$ needle was found to be preferable for the biopsy. The biopsies did not cause injuries to the bowel, ureters and large vessels. The biopsy was quickly performed, with a mean time required to perform the procedure of 6 minutes. Notably, it was possible to reach the inner myometrium (containing the junctional zone) in all the patients. The uterine specimens obtained by the biopsy allowed to prepare frozen sections, extract RNA and dissect single adenomyosis glands with laser dissection microscopy. In conclusion, this prospective study shows that biopsy of adenomyosis can be obtained in vivo. These preliminary data suggest that the biopsy can be safely performed. However, the study was not adequately powered to investigate this outcome and trials with larger sample size should be required in order to confirm the safety of this technique. In this study the biopsies were performed under general anesthesia; therefore, it was not possible to investigate if the procedure is painful. It seems likely that anesthesia or sedation is required similarly to what is performed for other procedures (such as oocyte pick-up). Adenomyosis specimens obtained by biopsies may allow to investigate the molecular pathway contributing to the development of the disease. This research may pave the way to the development of new drugs for the treatment of adenomyosis.

\section{Acknowledgments}

None.

\section{Footnote}

Conflicts of Interest: The authors have no conflicts of interest to declare.

Ethical Statement: The authors are accountable for all aspects of the work in ensuring that questions related to the accuracy or integrity of any part of the work are appropriately investigated and resolved.

\section{References}

1. Peric H, Fraser IS. The symptomatology of adenomyosis. Best Pract Res Clin Obstet Gynaecol 2006;20:547-55.

2. Ferrero S, Ragni N, Remorgida V. Deep dyspareunia: causes, treatments, and results. Curr Opin Obstet Gynecol 2008;20:394-9.

3. Leyendecker G, Bilgicyildirim A, Inacker M, et al. Adenomyosis and endometriosis. Re-visiting their association and further insights into the mechanisms of auto-traumatisation. An MRI study. Arch Gynecol Obstet 2015;291:917-32.

4. Scala C, Leone Roberti Maggiore U, Racca A, et al. Influence of adenomyosis on pregnancy and perinatal outcomes in women with endometriosis. Ultrasound Obstet Gynecol 2018;52:666-71.

5. Horton J, Sterrenburg M, Lane S, et al. Reproductive, 
obstetric, and perinatal outcomes of women with adenomyosis and endometriosis: a systematic review and meta-analysis. Hum Reprod Update 2019;25:592-632.

6. Mikos T, Lioupis M, Anthoulakis C, et al. The Outcome of Fertility-Sparing and Nonfertility-Sparing Surgery for the Treatment of Adenomyosis. A Systematic Review and Meta-analysis. J Minim Invasive Gynecol 2019. [Epub ahead of print].

7. Osada H. Uterine adenomyosis and adenomyoma: the surgical approach. Fertil Steril 2018;109:406-17.

8. Van den Bosch T, Dueholm M, Leone FP, et al. Terms, definitions and measurements to describe sonographic features of myometrium and uterine masses: a consensus opinion from the Morphological Uterus Sonographic Assessment (MUSA) group. Ultrasound Obstet Gynecol 2015;46:284-98.

9. Champaneria R, Abedin P, Daniels J, et al. Ultrasound scan and magnetic resonance imaging for the diagnosis of adenomyosis: systematic review comparing test accuracy. Acta Obstet Gynecol Scand 2010;89:1374-84.

10. Meredith SM, Sanchez-Ramos L, Kaunitz AM. Diagnostic accuracy of transvaginal sonography for the diagnosis of adenomyosis: systematic review and metaanalysis. Am J Obstet Gynecol 2009;201:107.e1-6.

11. Andres MP, Borrelli GM, Ribeiro J, et al. Transvaginal Ultrasound for the Diagnosis of Adenomyosis: Systematic Review and Meta-Analysis. J Minim Invasive Gynecol 2018;25:257-64.

12. Vannuccini S, Luisi S, Tosti C, et al. Role of medical therapy in the management of uterine adenomyosis. Fertil Steril 2018;109:398-405.

13. Ferrero S, Remorgida V, Venturini PL. Current pharmacotherapy for endometriosis. Expert Opin Pharmacother 2010;11:1123-34.

14. Ferrero S, Evangelisti G, Barra F. Current and emerging treatment options for endometriosis. Expert Opin Pharmacother 2018;19:1109-25.

15. Ferrero S, Barra F, Leone Roberti Maggiore U. Current and Emerging Therapeutics for the Management of

Cite this article as: Ferrero S, Scala C, Vellone VG, Biscaldi E, Barra F. Transvaginal ultrasound-guided biopsy of adenomyosis. Ann Transl Med 2019;7(Suppl 8):S341. doi: 10.21037/ atm.2019.09.94
Endometriosis. Drugs 2018;78:995-1012.

16. Kelekci S, Kelekci KH, Yilmaz B. Effects of levonorgestrelreleasing intrauterine system and T380A intrauterine copper device on dysmenorrhea and days of bleeding in women with and without adenomyosis. Contraception 2012;86:458-63.

17. Ozdegirmenci O, Kayikcioglu F, Akgul MA, et al. Comparison of levonorgestrel intrauterine system versus hysterectomy on efficacy and quality of life in patients with adenomyosis. Fertil Steril 2011;95:497-502.

18. Fawzy M, Mesbah Y. Comparison of dienogest versus triptorelin acetate in premenopausal women with adenomyosis: a prospective clinical trial. Arch Gynecol Obstet 2015;292:1267-71.

19. Hirata T, Izumi G, Takamura M, et al. Efficacy of dienogest in the treatment of symptomatic adenomyosis: a pilot study. Gynecol Endocrinol 2014;30:726-9.

20. Ferrero S, Camerini G, Menada MV, et al. Uterine adenomyosis in persistence of dysmenorrhea after surgical excision of pelvic endometriosis and colorectal resection. J Reprod Med 2009;54:366-72.

21. Goswami A, Khemani M, Logani KB, et al. Adenomyosis: diagnosis by hysteroscopic endomyometrial biopsy, correlation of incidence and severity with menorrhagia. J Obstet Gynaecol Res 1998;24:281-4.

22. Jeng CJ, Huang SH, Shen J, et al. Laparoscopy-guided myometrial biopsy in the definite diagnosis of diffuse adenomyosis. Hum Reprod 2007;22:2016-9.

23. Nam JH, Lyu GS. Abdominal ultrasound-guided transvaginal myometrial core needle biopsy for the definitive diagnosis of suspected adenomyosis in 1032 patients: a retrospective study. J Minim Invasive Gynecol 2015;22:395-402

24. Tellum T, Qvigstad E, Skovholt EK, et al. In Vivo Adenomyosis Tissue Sampling Using a Transvaginal Ultrasound-guided Core Biopsy Technique for Research Purposes: Safety, Feasibility, and Effectiveness. J Minim Invasive Gynecol 2019;26:1357-62. 